\title{
Entwicklungsperspektiven für das Europäische Parlament
}

von Udo Di Fabio

\section{Das Europäische Parlament in der unionalen Demokratie- architektur}

Das Europäische Parlament ist Teil des unionalen Institutionensystems. Die Abgeordneten werden seit dem Direktwahlakt des Jahres $1976^{1}$ nach nationalem Wahlrecht unmittelbar gewählt, weswegen seit 1979 nicht mehr von der mittelbar beschickten Versammlung, sondern vom Europaparlament die Rede ist. Die funktionale Deutung dieser Institution ist freilich schillernd. Für die einen ist das Europaparlament der eigentliche Ort demokratischer Legitimation, weil nur hier Unionsbürger direkt wählen. ${ }^{2}$ Ein europäisches Demokratiedefizit ${ }^{3}$ wird gleichgesetzt mit fehlender Entscheidungsmacht des Parlaments, weil die nur mittelbaren Legitimationsketten aus den Staaten hinein in den Rat und die Kommission als zu lang gelten, als zu stark mediatisiert und intransparent. ${ }^{4}$ Die Ausweitung der Kompetenzen des Parlaments wäre danach - ungeachtet der nur degressiv proportionalen Besetzung ${ }^{5}$ und des nicht staatsanalogen Aufbaus der EU ${ }^{6}$ - per se eine Minderung des Demokratiedefizits. Für fundamentale Kritiker dagegen, nicht nur die britische UKIP, ${ }^{7}$ ist das Europaparlament vor allem ein Ort europäi-

1 Beschluss und Akt zur Einführung allgemeiner unmittelbarer Wahlen der Abgeordneten des Europäischen Parlaments vom 20.09.1976 (BGBl. 1977 II, S. 733), zuletzt geändert durch Beschluss des Rates vom 25.06.2002 und 23.09.2002 (BGB1. 2003 II, S. 810).

2 Haltern, U.: Europarecht. Dogmatik im Kontext, Tübingen, ${ }^{2} 2007,135 \mathrm{ff}$.

3 Unter vielen zuletzt Franzius, C.: Demokratisierung der Europäischen Union, in: EuR, 6 (2013), 655-667; relativierend im Hinblick auf die Frage, ob ein solches Demokratiedefizit überhaupt vorliegt: Bergmann, J.: Handlexikon der Europäischen Union, Baden-Baden, ${ }^{4}$ 2012, Stichwort „Demokratiedefizit“.

4 Darstellung der diesbezüglichen Kritik vor allem am Rat bei Kirsch, A.: Demokratie und Legitimation in der Europäischen Union, Baden-Baden, 2008, 72f.

5 Art. 14 Abs. 2 UAbs. 1 Satz 3 EUV.

6 BVerfGE 123, 267 (365).

7 Vgl. die frühe Analyse in: Ford, R./Goodwin, M./Cutts, D.: Strategic Eurosceptics and polite xenophobes: Support for the United Kingdom Independence Party (UKIP) in the 2009 European Parliament elections, in: European Journal of Political Research, 51/2 (2012), 204-234. 
scher Selbstbezüglichkeiten, ohne echte Resonanz und Kontrolle durch die Wahlbürger, was das bestehende Demokratiedefizit eher verschärft als mindert.

Eine mittlere Linie zwischen diesen Positionen nimmt insofern das Bundesverfassungsgericht ein, das frühzeitig mit der Solange I-Entscheidung eine Parlamentarisierung des europäischen Institutionensystems für wünschenswert hielt ${ }^{8}$ und seit dem Maastricht-Urteil nüchtern eine doppelte Legitimation diagnostizierte: diejenige über die Mitgliedstaaten und zunehmend auch die über die Wahl der Abgeordneten des EU-Parlaments. ${ }^{9}$ Danach bestehen parallele (oder als dual bezeichnete ${ }^{10}$ ) Legitimationsstränge einerseits mittelbar von den Mitgliedstaaten in die Union hinein und andererseits unmittelbar durch Wahl der Abgeordneten des EU-Parlaments.

Das Selbstverständnis des Europäischen Parlaments selbst klingt ganz im erstgenannten Sinne manchmal wie die Forderung nach Parlamentssuprematie ${ }^{11}$, wenn etwa die intergouvernementalen Maßnahmen zur Eurostabilisierung seit 2010 kritisiert werden, obwohl das Budgetrecht der Staaten außerhalb der verpflichtenden Stabilitätskriterien nicht der Kompetenz der Union unterfällt. Das Parlament sieht jedenfalls - wer sollte es ihm verdenken - in jedem eigenen Kompetenzgewinn eine Minderung eines unterstellten strukturellen Demokratiedefizits.

\section{Stärkung des Parlaments nach dem Muster der staatsanalogen Entwicklungslogik}

Mit jedem Vertragsschritt von den Römischen Verträgen bis zum Lissabonvertrag wurden Versammlung und Parlament von einem peripheren, weil machtlosen Anhörungsgremium zu einem zentralen Organ der Legislative und der Kommissionskontrolle bis hinein in den Bereich politischer Initiativen, die eigentlich von der Kommission oder dem Europäischen Rat zu erwarten wären. ${ }^{12}$ Im entscheidenden

8 BVerfGE 37, 271 (280): „Sie [die Gemeinschaft] entbehrt noch eines unmittelbar demokratisch legitimierten, aus allgemeinen Wahlen hervorgegangenen Parlaments, das Gesetzgebungsbefugnisse besitzt und dem die zur Gesetzgebung zuständigen Gemeinschaftsorgane politisch voll verantwortlich sind $[\ldots] . “$

9 Als zweiter Legitimationsstrang neben die aus den Mitgliedstaaten tretend: BVerfGE 89, 155 (185f.).

10 Von Bogdandy, A.: Grundprinzipien, in: Ders./Bast, J.. (Hg.): Europäisches Verfassungsrecht, Berlin, ${ }^{2} 2009$, 13-72, hier 62ff.; Möllers, C./von Achenbach, J.: Die Mitwirkung des Europäischen Parlaments an der abgeleiteten Rechtsetzung der Europäischen Kommission nach dem Lissabonner Vertrag, in: EuR, 1 (2011), 39-47.

11 Zum englischen Verständnis insbesondere im Hinblick auf die europäische Integration: Sydow, G.: Parlamentssuprematie und Rule of Law. Britische Verfassungsreformen im Spannungsfeld von Westminster Parliament, Common-Law-Gerichten und europäischen Einflüssen, Tübingen, 2005.

12 Schorkopf, F.: Der Europäische Weg. Grundlagen der Europäischen Union, Tübingen, 2010, $143 \mathrm{ff}$. 
Bereich der Rechtsetzung ist das „ordentliche Gesetzgebungsverfahren“ seit Lissabon der Regelfall (Art. 289 Abs. 1 AEUV), d.h. ohne die Zustimmung des Parlaments kann eine Verordnung oder Richtlinie nicht ergehen. ${ }^{13}$ Daraus folgt nicht etwa nur eine Vetoposition, sondern eine echte politische Gestaltungsfunktion, die etwa im informellen Trilog ${ }^{14}$ der an der Gesetzgebung beteiligten Organe zur Geltung gelangt. Der Rat kann selbst den Beschluss über den Abschluss eines völkerrechtlichen Vertrags in wichtigen Fällen erst nach Zustimmung des Europäischen Parlaments erlassen. ${ }^{15} \mathrm{Zu}$ diesen Bereichen zählt erstmals die gemeinsame Handelspolitik $^{16}$ oder die Rahmenfestlegung für Maßnahmen der humanitären Hilfe, einschließlich der Entsendung eines Europäischen Freiwilligenkorps. ${ }^{17}$ Daneben entscheidet das Europäische Parlament mit dem Rat gleichberechtigt über den Haushaltsentwurf (Art. 314 AEUV).

Eine weitere Stärkung des Parlaments nahm der Lissabon-Vertrag bekanntlich hinsichtlich der Kreation und Kontrolle der Kommission vor. Der Kommissionspräsident wird nach der Wahl 2014 nicht mehr vom Europäischen Rat nach Zustimmung des Europäischen Parlaments benannt, ${ }^{18}$ sondern dann vom neu konstituierten Europäischen Parlament mit der Mehrheit seiner Mitglieder gewählt (Art. 17 Abs. 7 EUV-Lissabon). Findet der von dem Europäischen Rat vorgeschlagene Kandidat keine Mehrheit im Europäischen Parlament, muss der Europäische Rat dem Europäischen Parlament innerhalb eines Monats einen neuen Kandidaten vorschlagen. Aus dieser Änderung im Kreationsverfahren der Kommission nehmen die größeren Parteienzusammenschlüsse das Recht in Anspruch, mit Spitzenkandidaten in den Europawahlkampf zu gehen, die sie als Kommissionspräsidenten durchsetzen wollen, wobei die beiden aussichtsreichsten Kandidaten - der Logik der parallelen Entscheidungsstruktur folgend - informell das

13 Nach CEPS/EGMONT/EPC: The Treaty of Lisbon. Implementing the Institutional Innovations, 2007, 7, wurden 30 bestehende Rechtsgrundlagen geändert, um ihren Gebrauch dem ordentlichen Gesetzgebungsverfahren zu unterwerfen, und 13 neue, bereits mit dem ordentlichen Gesetzgebungsverfahren verknüpfte Rechtsgrundlagen eingeführt. Siehe auch BTDrucks 16/8300, 146ff., Tabelle 2: Übergang in das ordentliche Gesetzgebungsverfahren.

14 Gemeinsame Erklärung zu den praktischen Modalitäten des neuen Mitentscheidungsverfahrens (Artikel 251 EG-Vertrag), AB1. Nr. C 145 vom 30.06.2007, 5, Dok. Nr. 32007C0630(01).

15 Die starke Stellung des Parlaments besteht in Bereichen, für die entweder das ordentliche Gesetzgebungsverfahren oder, wenn die Zustimmung des Europäischen Parlaments erforderlich ist, das besondere Gesetzgebungsverfahren gilt (Art. 218 Abs. 6 UAbs. 2 Buchstabe a Nr. v AEUV). Art. 300 Abs. 3 EGV sah beim Abschluss von völkerrechtlichen Verträgen grundsätzlich nur eine Anhörung des Europäischen Parlaments vor.

16 Art. 218 Abs. 1, Art. 207 Abs. 3, Art. 218 Abs. 6 UAbs. 2 Buchstabe a AEUV.

17 Art. 214 Abs. 2 und 3 AEUV sowie Art. 214 Abs. 5 AEUV.

18 So noch die Regelung des Art. 214 Abs. 2 EGV. 
grundsätzliche Plazet von Staats- und Regierungschefs eingeholt haben (dürften). Da die Kommission durch ihr exklusives Gesetzesinitiativrecht konzeptionell stark ist, über nicht unwesentliche Verwaltungsmacht verfügt und zudem mit dem Lissabonner Vertrag abgeleitete Rechtsetzungsbefugnisse erhalten hat, ${ }^{19}$ kommt es nicht nur auf die Kreation der Kommission als Einmalvorgang an, sondern auch auf die stete kontrollierende parlamentarische Beobachtung. Dem Parlament wird es dabei um die Wahrung eigener Zuständigkeiten, aber auch um politisch inhaltliche Kontrolle gehen, damit die Verantwortung gegenüber den Wählern von den Abgeordneten wahrgenommen werden kann.

\section{Das Parlament als Repräsentationsorgan in der dualen Struktur des Verbundsystems}

Das europäische Parlament repräsentiert nicht das europäische Volk, das es als völkerrechtlichen und staatsrechtlichen Demos nicht gibt. ${ }^{20}$ Das Europäische Parlament besteht zwar aus Vertretern der „Unionsbürgerinnen und Unionsbürger“ (Art. 14 Abs. 2 UAbs. 1 Satz 1 EUV) und nicht mehr wie zuvor ,der Völker der in der Gemeinschaft zusammengeschlossenen Staaten“..21 Daraus darf aber keine Singularisierung des demokratischen Legitimationssubjekts geschlossen werden, denn die Unionsbürger sind dies nur vermittelt über ihre Staatsbürgerschaft in einem Mitgliedstaat der Union (Art. 20 Abs. 1 Satz 2 AEUV), und vor allem ist das Europaparlament wegen der nationalen Kontingentierung der Sitzverteilung immer noch ein Verbundparlament, das Grundsätze der Bürgerrepräsentation und der Staatenvertretung miteinander kombiniert. Deshalb muss hier der Grundsatz der Wahlgleichheit im Zählwert der Stimme (,one man, one vo$\left.t e^{6}\right)$ nicht gelten, ja streng genommen darf er nicht einmal gelten, ohne den Charakter eines Verbundes von Staaten konzeptionswidrig zum Bundesstaat hin zu verschieben. $^{22}$ Der Vertrag von Lissabon hat deshalb den bis dahin immerhin

19 Dazu näher Möllers, C./von Achenbach, J., a.a.O.

20 Anders Peters, A.: Elemente einer Theorie der Verfassung Europas, Berlin, 2001, 657ff., die den einzelnen zuerst entstaatlicht (individualisiert) und ihn dann wieder in der Union rekonstruiert. Zum Repräsentationsprinzip Kaufmann, M.: Europäische Integration und Demokratieprinzip, Baden-Baden, 1997, 69f.; zu inhärenten Gefährdungslagen des Prinzips: Steinberg, R.: Die Repräsentation des Volkes. Menschenbild und demokratisches Regierungssystem, Baden-Baden, 2013, 163ff.; Huber, P.M.: Selbstbestimmung in Europa, in: ZSE, 11/4 (2013), 484-505, hier 496f.

21 So noch Art. 189 Abs. 1 EGV.

22 Das Verbundsystem ist eine auf Dauer angelegte Verbindung von Staaten, die völkerrechtlich souverän bleiben, aber sich unter Geltung verbindlichen Vertragsrechts nach dem Prinzip der begrenzten Einzelermächtigung einer von ihnen gemeinsam bestimmten autonomen Herrschaftsgewalt unterwerfen. Siehe BVerfGE 123, 267 (348). 
registrierbaren Trend zur allmählichen Verstärkung der Stimmengleichheit eher wieder in die andere Richtung gedreht und an die Stelle des subkutanen Näherungsziels sukzessiv anzustrebender Gleichheit nunmehr den Grundsatz der degressiven Proportionalität gesetzt. Danach muss lediglich das Verhältnis zwischen der Bevölkerung und der Zahl von Sitzen jedes Mitgliedstaats in Abhängigkeit von seiner jeweiligen Bevölkerung variieren, jedoch dürfen bei vorgegebener Sitzzahl (750 Vertreter) bestimmte Untergrenzen für die Staatenkontingente (6 Mandate mindestens) und bestimmte Obergrenzen (96 Mandate höchstens) nicht verletzt werden (Art. 14 Abs. 2 UAbs. 1 Satz 2 bis 4 AEUV). ${ }^{23}$ Schon das Korsett der degressiven Proportionalität lässt es nicht zu, von einer bundesstaatlichen Volksvertretung im Singular zu sprechen, denn die so zustande gekommenen Beschlüsse des Parlaments sind nicht notwendig auf eine Mehrheit der Wählerstimmen zurückzuführen; der Beschluss kann also nach der Vorstellung der parlamentarischen Volksrepräsentation nicht verlässlich als Mehrheitsentscheidung des Volkes rubriziert werden.

Ein solch ersichtlicher Befund - der im Lissabon-Urteil des BVerfG deskriptiv und nicht pejorativ zu finden ist ${ }^{24}$ und gleichwohl wegen angeblicher Aversion gegen das Europäische Parlament für Kritik gesorgt hat - nimmt dem Europaparlament indes nichts von seiner Bedeutung im unionalen Organsystem, gerade unter dem Gesichtspunkt der unmittelbaren Quelle demokratischer Legitimation. Allerdings kann das Parlament nicht so suprematisch auftreten wie das staatliche Repräsentationsorgan des Volkes: Es ist ein inzwischen sehr starker Akteur in dem verhandlungsaffinen kooperativen Verbundsystem, kann aber nicht ein Mandat des ,overruling“ insbesondere gegenüber dem Rat in Anspruch nehmen.

In der dualen Herrschaftsstruktur des europäischen Verbundsystems ${ }^{25}$ hat sich die Rolle des Parlaments auch institutionell innovativ gewandelt und sollte deshalb nicht allein im Blick auf eine immer wieder anzutreffende allein staatsanaloge Entwicklungsrichtung begriffen werden. ${ }^{26}$ Das Europäische Parlament ist

23 Vor Lissabon hatte Art. 190 Abs. 2 EGV ausdeutungsfähig im Sinne allmählicher Gleichheitsannäherung bestimmt, dass die Sitzverteilung im Europäischen Parlament eine ,angemessene Vertretung der Völker der in der Gemeinschaft zusammengeschlossenen Staaten“ voraussetzt.

24 BVerfGE 123, 267 (371ff.).

25 Siehe oben Fn. 22.

26 Das spielte auch eine Rolle bei den durch abweichende Meinungen dokumentierten Meinungsverschiedenheiten innerhalb des Zweiten Senats des BVerfG zu Sperrklauseln betreffend die Wahl der deutschen Vertreter im Europaparlament, siehe BVerfGE 129, 300 (335ff.) einerseits und die Mindermeinung in BVerfGE 129, 300 (353ff.) andererseits. 
nicht nur Legislativ- und Kreationsorgan, es besitzt auch ein konzeptionelles politisches Handlungs- und das heißt immer auch Verhandlungsmandat. Der außenpolitische Ausschuss des Parlaments beispielsweise spiegelt nicht nur als Kontrolleur der Exekutive das Handeln der Außenbeauftragten, die unter Verbundbedingungen anders agieren muss als staatlich gewohnt. Der Ausschuss ist zugleich der Ort, an dem ein außenpolitisches Unionsinteresse staatenübergreifend formuliert werden kann und als Position aktiv und initiativ deutlich gemacht wird. Ähnliches dürfte bei der gemeinsamen Handelspolitik oder der Industriepolitik der Fall sein.

\section{Perspektiven des Europaparlaments}

Das neue Europaparlament wird bei verstärkter Ausstattung mit Kompetenzen künftig in einem deutlich schwierigeren Umfeld agieren, und das nicht etwa nur, weil die zunehmenden Erosionen ${ }^{27}$ in den politischen Kulturen der Mitgliedstaaten auf Wahl und Zusammensetzung des Parlaments durchschlagen. Die Rahmenbedingungen der Union haben sich seit der Weltfinanzkrise verschlechtert, die Probleme sind jedenfalls erheblich anspruchsvoller geworden. Die europäische Schuldenkrise ist solange nicht gelöst, wie die Staaten nicht mit höherer Wettbewerbsfähigkeit einen gesunden Wachstumspfad betreten und die Rückführung der Defizite und Schuldenstände auf das rechtlich verbindliche Maß aussteht. Politische Fehler der Mitgliedstaaten schlagen auf das europäische Institutionensystem durch, ohne dass von Brüssel aus autoritativ entschieden und korrigiert werden könnte. Die Kontrolle des Finanzsystems in der Bankenunion schiebt die EZB in eine der geldpolitischen Funktion fremde operative Aufsichtsrolle, ${ }^{28}$ weil bei der Bankenregulation politisch und rechtlich weder der Weg in die Zentralisierung noch in die Re-Nationalisierung eröffnet ist. Die Aufgabe der Koordinierung der Wirtschaftspolitiken ist anspruchsvoll. Eine Wirtschaftsregierung ist weder von den Verträgen vorgesehen noch überhaupt sinnvoll angesichts der Heterogenität der volkswirtschaftlichen Bedingungen in den Mitgliedstaaten. Gleichwohl zeigen die Einrichtungen des EFS und des ESM, die wichtige Arbeit der sogenannten Troika, die übernommene Rolle der EZB und der Fiskalpakt,

27 Di Fabio, U.: Erosion des Staates, in: Bredekamp, H./Føllesdal, D./Di Fabio, U. (Hg.): Transzendenzen des Realen, Bonn, 2013, 165-188; Schliesky, U.: Souveränität und Legitimität von Herrschaftsgewalt. Die Weiterentwicklung von Begriffen der Staatslehre und des Staatsrechts im europäischen Mehrebenensystem, Tübingen, 2004, $311 \mathrm{f}$.

28 Di Fabio, U.: Die Zukunft einer stabilen Wirtschafts- und Währungsunion. Verfassungs- sowie europarechtliche Grenzen und Möglichkeiten. Hg. v. der Stiftung Familienunternehmen, München, 2013, 55ff. 
dass Wege zur Konsolidierung bestehen. ${ }^{29}$ Das Europaparlament sollte hier nicht nur kritisch die intergouvernementale Methode beklagen und auf eigene Kompetenzen pochen: Die Haushalts-, Wirtschafts- und Sozialpolitik der Staaten liegt nicht in der primären Zuständigkeit der Union, sondern der Mitgliedstaaten. Auch das Europaparlament sollte hier mehr Gespür für die Notwendigkeiten der vertraglich fixierten Kompetenzabgrenzung zeigen. ${ }^{30}$ Wenn die Mitglieder der Währungsunion flankierend die europäische Architektur von außen stützen und durch völkerrechtliche Bindung einen Beitrag dazu leisten, eine akute Krise zu überwinden und nationale Politikräume wieder näher an das Recht heranzuführen, sollte dies begrüßt und nicht getadelt werden. Hier ist kein Rückfall in eine Kultur der europäischen Staatenkonferenz zu befürchten, sondern eine institutionelle Elastizität zu diagnostizieren, ${ }^{31}$ die allerdings nicht auf Dauer deformierend, sondern stützend für die Einhaltung des Vertragsrechts werden darf. Man könnte sich sogar vorstellen, dass das künftige Europaparlament seine Kontrolle der Kommission auch insofern intensiviert, als kritisch nachgefragt wird, ob die Kommission genug tut, um die für die Funktionsfähigkeit der Währungsunion entscheidende Einhaltung der Stabilitätskriterien wirksamer zu überwachen und ein sorgsames Auge auf Schleichwege aus der Knappheit öffentlicher Mittel zu haben. Der besondere Charakter des nunmehr auf fünf Jahre neu mandatierten Verbundparlamentes könnte auch darin liegen, nicht jeder Stimmung nachzugehen, wie das in nationalen Politikräumen manchmal der Fall ist, sondern die rechtlichen, wirtschaftlichen und technologischen Grundlagen der Union als Hüter europäischen Rechts und eines freiheitsgerechten Gemeinwohls noch deutlicher zu wahren. Damit wäre das Europäische Parlament jedenfalls in seiner konstruktiven Mehrheit möglicherweise ein Gegengewicht zu populistischen Ausgabenprogrammen und Wahlversprechungen, wenn diese nicht solide finanziert sind oder gegen vernünftige Grundsätze der Generationenverantwortung verstoßen.

Auch die Außen- und Sicherheitspolitik der Union verlangt nach sinnvoller und vertragsentsprechender Einflussnahme des Europäischen Parlaments. Die EU ist mit ihrer Ausdehnung ein Stück weit Opfer ihres Erfolges geworden. Der Beitritt

29 Die Heterogenität darf dabei nicht nur als Hemmnis verstanden werden, das wegreguliert werden könnte, siehe etwa Gros, D.: How to deal with macroeconomic imbalances in the Euro area, in: CEPS Policy Brief, 266 (April 2012).

30 Huber, P.M., a.a.O., 502.

31 Kritisch insofern etwa Kadelbach, S.: Lehren aus der Finanzkrise - Ein Vorschlag zur Reform der Politischen Institutionen der Europäischen Union, in: EuR, 5. (2013), 489-503, hier $497 f$. 
zur EU bedeutet für Transformationsstaaten auf dem Balkan oder in Osteuropa, denen es bislang an Möglichkeiten zu demokratischer Selbstbestimmung, rechtsstaatlichen Strukturen und marktwirtschaftlicher Wettbewerbsfähigkeit fehlte, in eine Gemeinschaft einzutreten, die all das verlangt und befördert. Aber die Erweiterungsdynamik hat nicht nur die innere Funktionsfähigkeit der EU belastet und ihre Entwicklungsrichtung modifiziert, ${ }^{32}$ sie ruft inzwischen auch politische Gegenkräfte und Widerstände hervor, wenn man an die Ukraine oder die Beziehungen zum Nahen Osten oder dem nordafrikanischen Grenzraum denkt. Die sanfte Macht der offenen Marktwirtschaft, die durch den Schutzschild einer verbündeten Supermacht flankiert wird, stößt auf Gegenspieler und Regionen, die an Gewalt als Mittel der Politik festhalten. ${ }^{33}$ Die Lösung politischer Machtgegensätze und die Bekämpfung des alten Staatenantagonismus durch funktionale Wirtschaftseinigung im Binnenmarkt war für das westliche Nachkriegseuropa die große Erfindung, die nach 1990 noch einmal erheblich ausgedehnt wurde, aber nicht notwendig jeden Machtspieler an der Peripherie zu überzeugen vermag. Was kann man daraus folgern? Mehr Selbstbeschränkung oder mehr Machtentfaltung? Für manche ist im Sinne der Machtkonzentration eine europäische Armee mit annähernder Schlagkraft der US-Streitkräfte ein dringlicher werdendes Integrationsziel. Andere wären bereits über eine harmonisierte Außenpolitik glücklich. Doch nicht Supermachtsträume und die Ideologie der großen Zahl sollten die Ratgeber sein, sondern kühler Pragmatismus in der Kunst des Möglichen. Auch hier bedarf es eines Parlaments der politischen Reife, das nicht institutionellen Blütenträumen nachjagt, sondern die Regierungen der Mitgliedstaaten mit dem europäischen Gemeinwohl stärker konfrontiert. Manche Staaten, wie Polen oder die baltischen Republiken, vertrauen auf die NATO und die USA mehr als auf eine zukunftsferne wirksame gesamteuropäische Verteidigung. Die Außenpolitik Europas muss stärker koordiniert werden, aber auch im festen transatlantischen Bündnis bleiben. In der NATO sind die nationalen Verteidigungsbeiträge längst integriert und müssen auf neue Bedrohungslagen hin wie immer in der Geschichte des Bündnisses ${ }^{34}$ - fortgeschrieben werden. Nicht Zentralisierung ohne politische Unterstützung der Bevölkerung ist das Allheil-

32 Brasche, U.: Europäische Integration: Wirtschaft, Erweiterung und regionale Effekte, München, ${ }^{3} 2013$, $398 \mathrm{ff}$.

33 Das wurde bereits im Fall Griechenland vs. Türkei als Problem gesehen, das sich verglichen mit der gegenwärtigen Lage eher als relativ gut zu bewältigen erwiesen hat. Siehe Rumelili, B.: Transforming conflicts on EU borders: The case of Greek-Turkish relations, in: Journal of Common Market Studies, 45/1 (2007), 105-126.

34 BVerfGE 104, $151 \mathrm{ff}$. 
mittel gegen die angeblichen Gebrechen einer Zersplitterung der Kräfte. Erforderlich ist wachsendes Verständnis für notwendige und womöglich auch wieder steigende Beiträge aller Mitglieder von EU und NATO zu einer effektiven transatlantischen Verteidigung, aber auch ein umsichtigerer Blick auf Reaktionen, die Assoziierungsabkommen im Grenzraum der EU auslösen können. 